Tropical Journal of Pharmaceutical Research, August 2009; 8 (4): 361-369

(C) Pharmacotherapy Group,

Faculty of Pharmacy, University of Benin,

Benin City, 300001 Nigeria.

All rights reserved.

Research Article

Available online at http://www.tjpr.org

\title{
Polymeric Recrystallized Agglomerates of Cefuroxime Axetil Prepared by Emulsion Solvent Diffusion Technique
}

\author{
VB Yadav ${ }^{\star 1}$ and AV Yadav ${ }^{2}$ \\ ${ }^{1}$ Govt.College of Pharmacy, Vidyanagar, Karad, Dist-Satara (MS), ${ }^{2}$ Goa College of Pharmacy, 18th June
} Road, Panaji, Goa, India

\begin{abstract}
Purpose: To study the effect of different polymers on the solubility and dissolution rate of cefuroxime axetil (CFU) prepared by emulsion solvent diffusion (ESD) technique.

Methods: The ESD technique employed involved three test solvents: first, substance dissolution medium-good solvent (acetone); second, partial dissolution medium for the substance-bridging liquid (dichloromethane), and third, immiscible with the substance-poor solvent (distilled water). The pure CFU and the prepared agglomerates were characterized in terms of production yield, drug content, solubility, in vitro release profile, flowability, density, wettability, as well as by thin layer chromatography (TLC), differential scanning calorimetry (DSC), x-ray diffraction (XRD), Fourier transforms infra red spectroscopy (FTIR) and stability test.

Results: DSC showed a decrease in the melting enthalpy indicating disorder in the crystalline content. $X R D$ also indicated changes in crystallinity, FTIR revealed that there were no chemical changes in the recrystallized agglomerates while dissolution data demonstrated a marked increase in the dissolution rate (>55\% in $45 \mathrm{~min})$ compared with the pure drug (35\% in $45 \mathrm{~min})$. The improvement in the dissolution rate of CFU from optimized crystal formulation was attributed to the wetting effect of the polymers, change in drug crystallinity, altered surface morphology and micronization. The recrystallized agglomerates also exhibited higher wettability and flowability.

Conclusion: The optimised recrystallized agglomerates exhibited good solubility, wettability, dissolution rate and other physicochemical properties compared to the unmodified CFU.
\end{abstract}

Key words: Cefuroxime axetil; Recrystallized agglomerates; Solubility; Dissolution; Physicochemical properties. 


\section{INTRODUCTION}

Aqueous solubility and dissolution are two of the crucial factors influencing drug absorption from the gastrointestinal tract (GIT). However, many newly discovered compounds exhibit low oral bioavailability. The oral route is convenient for administration of drugs compared to other routes; therefore, in developing novel oral drug delivery systems, different strategies are employed to increase the solubility and dissolution of liphophilic drugs, e.g., particle size reduction, solid dispersion, complexation with cyclodextrins, etc.

Several methods of reducing particle size have been suggested. They include physical methods such as milling and grinding ${ }^{1}$, but it is difficult to obtain uniform fine particles and control the particle size of the drug. Microcrystallization of hydrophobic drugs is a technique to overcome these problems ${ }^{2}$. The process is usually used to obtain small particles by the disruption of large crystals Dissolution rate and bioavailability improvement by micronization of sparingly water-soluble drugs entail using jar mills and fluid energy mills ${ }^{3}$. However, the micronization process is extremely inefficient due to the involvement of high energy nanoparticles and physical and/or chemical instability caused by disruptions in the crystal lattice ${ }^{4-6}$. Spray-drying of a drug solution is another way to produce micron-sized spherical particles. Spray-dried drugs, which are amorphous, show a smaller and more homogeneous particle size than mechanically micronized drugs ${ }^{7,8}$. However, as a fine particle fraction, even spray-dried amorphous drugs show only incomplete dispersion ${ }^{9}$.

The spherical crystallization technique has already been successfully applied to improve the micromeritic properties of acebutolol hydrochloride ${ }^{10}$. In the most common case, this technique is reputed to improve the wettability and dissolution rate of different drugs $^{11-14}$. Some drugs have also been recrystallized by the spherical agglomeration technique using polymeric materials to modify their release ${ }^{15,16}$. There are two main methods of spherical crystallization: spherical agglomeration (SA) and emulsion solvent diffusion (ESD).

Cefuroxime is the first commercially available second generation cephalosporin which is active against a wide range of Gram-positive and Gram-negative organisms and is resistant to most $\beta$-lactamases. It is a semisynthetic cephalosporin obtained from the 7cephalosporanic acid nucleus of cephalosporin $^{17}$. Cefuroxime axetil (CFU) is the acetoxyethyl ester of the cephalosporin, cefuroxime (a prodrug of cefuroxime). It is a Biopharmaceutics Classification System (BCS) class II drug and has only 55\% absolute oral bioavailability due to poor aqueous solubility. Since it is slightly soluble in water and can form a thicker gel on contact with aqueous medium, therefore, it shows poor dissolution and consequently, low bioavailability in the gastrointestinal tract ${ }^{18}$.

In this work, an attempt has been made to enhance the solubility, dissolution rate and other physicochemical properties of CFU by an emulsion solvent diffusion (EDS) method. The method employs three solvents: (i) substance dissolution medium (good solvent), (ii) partially dissolution medium for the substance (bridging liquid), and (iii) one that is immiscible with the substance (poor solvent). ESD is the particle engineering technique by which crystallization and agglomeration can be carried out simultaneously in one step to transform crystals directly into a compacted spherical form $^{19}$. This particle design technique has emerged as one of the areas of research interest in pharmaceutical manufacturing and has gained considerable attention due to the fact that crystal habit (form, surface, size and particle size distribution) can be modified during the crystallization process. Consequently, certain micrometric properties (bulk density, flow property, compactability) and physicochemical characteristics 
(solubility, dissolution rate, bioavailability and

\section{EXPERIMENTAL}

\section{Materials}

Cefuroxime axetil was received as a gift from Okasa Pharma (Satara, India). Methocel (HPMC, E-5) and hydroxypropyl cellulose were supplied by Colorcon, (Goa, India). Glyceryl monostearte was procured from Alembic (Vadodara, India) while Eudagit RLPO was obtained from Degussa India Pvt. Ltd Research Centre (Mumbai, India). Acetone and dichloromethane were purchased from S.D. Fine Chemicals (Mumbai, India).

\section{Preparation of cefuroxime axetil agglome- rates}

All spherical agglomerates were obtained by the emulsion solvent diffusion (ESD) method at room temperature using distilled water (as external phase and poor solvent). The internal phase contained a good solvent (acetone) and a bridging liquid (dichloromethane) with cefuroxime axetil (CFU, $1 \mathrm{~g}$ ), $0.25 \mathrm{~g}$ of polymer (either Eudragit RLPO, hydroxypropyl cellulose (HPC) or hydroxypropyl methylcellulose (HPMC)) mixed separately with glyceryl monostearate $(0.25 \mathrm{~g})$. The agglomerates were coded CFURLPO, CFU-HPC and CFU-HPMC accordingly.

In the ESD method, the drug, and polymer and glyceryl monostearate were co-dissolved in an organic solvent mixture of dichloromethane $(5 \mathrm{ml})$ and acetone $(5 \mathrm{ml})$. The drug-polymer solution was slowly added to the external phase $(150 \mathrm{ml})$ containing $0.25 \%$ PVA under agitation at $1000 \mathrm{rpm}$. The system was stirred continuously for about 12 h. As the good solvent diffused into the poor solvent, droplets gradually solidified and formed spherical agglomerates. The system was filtered through Whatman filter paper no. 42 under the vacuum to separate the spherical agglomerates. The resultant stability) can also be modified. product was washed with distilled water and dried in a hot air oven at $40{ }^{\circ} \mathrm{C}$ for $12 \mathrm{~h}$.

\section{Yield and drug content}

The dried agglomerates were weighed and the production yield calculated. Each agglomerate $(300 \mathrm{mg}$ ) was powdered, from which a sample equivalent to $100 \mathrm{mg} \mathrm{CFU}$ was weighed and extracted with three portions of $50 \mathrm{~mL} 0.07 \mathrm{~N} \mathrm{HCl}$. Each portion was filtered through a G-4 sintered glass filter and volume was adjusted to $100 \mathrm{~mL}$ with the same extraction medium. After sufficient dilution with $0.07 \mathrm{~N} \mathrm{HCl}$, samples were analyzed spectrophotometrically (Pharma Spec 1700, Shimadzu Corporation, Kyoto, Japan) at $281 \mathrm{~nm}$ and CFU content was calculated. The test was performed in triplicate.

\section{Saturation solubility studies}

Saturation solubility studies were carried out using deionized water as a solvent. Excessive quantity (sample $150 \mathrm{mg}$ ) of sample (pure drug or agglomerate) was taken in six screw capped test tubes with fixed volume $(10 \mathrm{ml})$ of deionized water. The resultant suspension was shaken at room temperature in an incubator shaker at 100 rpm for $24 \mathrm{~h}$ after which samples were withdrawn and filtered through $0.2 \mu$ filters (Ultipor ${ }^{\circledR} \mathrm{N}_{66}$, Pall Life sciences, Mumbai, India). The filtrate was suitably diluted with $0.07 \mathrm{~N} \mathrm{HCl}$ and analyzed at $281 \mathrm{~nm}$ by UV/VIS spectrophotometry. Triplicate measurements were made.

\section{In vitro drug release study}

The release rate of cefuroxime axetil and the agglomerates (sample equivalent to $100 \mathrm{mg}$ drug) were performed in $900 \mathrm{ml}$ of $0.07 \mathrm{~N} \mathrm{HCl}$ using USP Apparatus II (Disso 2000, Lab India Pvt. Ltd, Mumbai, India) set at $37 \pm 0.2^{\circ} \mathrm{C}$ $(\mathrm{n}=6)$ and rotated at $55 \mathrm{rpm}$. Samples $(5 \mathrm{ml})$ were withdrawn at regular intervals and replaced with the same volume of fresh 
dissolution medium to maintain sink conditions. The samples were suitably diluted with the dissolution medium and drug content assayed spectrophotometrically at $281 \mathrm{~nm}$ (Pharma Spec 1700, Shimadzu Corporation, Kyoto, Japan).

\section{Measurement of flowability and density}

The loose bulk density (LBD) and tapped bulk densities (TBD) were determined by using a density measuring apparatus (Serwell, Bangalore, India). An amount of the sample $(5 \mathrm{~g})$ was placed in a measuring cylinder and the volume (bulk volume) was measured after applying three taps. Tapped density was measured as per USP using the procedure described by Lafaver ${ }^{20}$. A quantity $(20 \mathrm{~g})$ of the material was passed through a $1 \mathrm{~mm}$ aperture sieve and transferred to a $100 \mathrm{~mL}$ graduated cylinder. The surface was carefully leveled out compacting the material. The unsettled apparent volume was noted. .The cylinder was tapped at a rate of 300 drops/min over a fixed drop distance of $14 \pm 2 \mathrm{~mm}$. After the first 500 drops, the volume of the material in the cylinder was measured. Further tapping (750 and then 1250 drops successively) was applied was applied until the difference between two volumes following successive tapping was less than $2.0 \%$. This final volume was taken as the tapped volume. Bulk and tapped densities, Carr's index (\%) and Hausner's ratio were calculated as in Eqs. 1 - 4. Angle of repose of the materials were assessed by the fixed funnel method and computed as in Eq. 5. The results presented are the means of three determinations each.

$$
\begin{aligned}
& \text { Bulk density }(\sigma b)=\text { Weight } / \text { Bulk volume ... } \\
& \text { Tapped density }(\sigma t)=\text { Weight } / \text { Tapped volume ... } \\
& \text { Carr's Index }=[(\sigma t-\sigma b) / \sigma t] \times 100 \ldots . . \\
& \text { Hausner ratio }=(\sigma t /(\sigma b) \ldots \ldots \ldots \ldots \ldots \\
& \text { Angle of repose }(\theta)=\operatorname{Tan}^{-1}(h / r) \ldots \ldots \ldots . . .
\end{aligned}
$$

\section{Wettability study}

Powder bed hydrophilicity test was carried out to assess the wettability of the agglomerates by placing the sample $(2 \mathrm{~g})$ in sintered glass tube to form a bed in the glass tube on which methylene blue crystals $(\approx 100$ $\mathrm{mg}$ ) were placed. The tube was brought into contact with the surface of water and the time taken for water to rise by capillary movement to dissolve methylene blue crystals was noted. The shortest time corresponds to the most wettable sample. The test was performed in triplicate.

\section{Differential scanning calorimetric (DSC) studies}

Differential scanning calorimetric (DSC) analysis of the samples was carried out with a DSC analyzer (model TA-60, Shimadzu Corporation, Kyoto, Japan). A sample (3-7 $\mathrm{mg}$ ) was sealed in an aluminum pan with a perforated lid and heated under nitrogen atmosphere at a heating rate of $10{ }^{\circ} \mathrm{C} / \mathrm{min}$ over the temperature range of $20-210{ }^{\circ} \mathrm{C}$. The thermograms were obtained and recorded.

\section{X-ray powder diffraction (XRPD) studies}

The XRPD patterns of the samples were monitored with an x-ray diffractometer (Philips PW 1729, Analytical XRD, Holland) using $\mathrm{Ni}$ filtered $\mathrm{CuK}(\alpha)$ radiation (intensity ratio $\left.\left(\alpha_{1} / \alpha_{2}\right): 0.500\right)$, voltage of $40 \mathrm{KV}$, current of $30 \mathrm{~mA}$ and receiving slit of 0.2 inches. The samples were analyzed over $2 \theta$ range of $5.010-39.990^{\circ}$ with scanning step size of $0.020^{\circ}(2 \theta)$ and scan step time of one second. To minimize the effect of particle size on preferred orientation, all the samples were first passed through sieve no. $120(125 \mu \mathrm{m})$ and collected on sieve no. $240(62.5 \mu \mathrm{m})$.

\section{Fourier transforms infra red spectroscopy (FTIR) studies}

The FT-IR spectra of the agglomerates and the pure drug (embedded in potassium 
bromide pellet) were recorded on Shimadzu FTIR - 8400 spectrophotometer (Shimadzu Corporation, Kyoto, Japan). Background spectrum was obtained under identical conditions. Each spectrum was derived from single average scans collected in the region $400-4000 \mathrm{~cm}^{-1}$ at a spectral resolution of 2 $\mathrm{cm}^{-2}$ and rationed against background interferogram. The spectra were analyzed by the in-built software.

\section{Statistical analysis}

The data was analyzed using GraphPad InState Demo and Microsoft Excel 2003. One-way analysis of variance (ANOVA) and Dunnett Multiple Comparisons Test were used test the statistical significance of the data. $P<0.0001$ was considered extremely significant. Variation among column means was significantly greater than expected by chance. If the value of $q$ is greater than 2.880 then the $P$ value is less than 0.05 .

\section{RESULTS}

The physicochemical and other properties of the CFU and its agglomerates are listed in Table 1. The yield of the recrystallized agglomerates was between 88 and $92 \%$ while drug content was in the range $93-96 \%$. The aqueous solubility of CFU in the agglomerates was between 1.33 and 1.59 $\mathrm{mg} / \mathrm{ml}$ compared to $0.3 \mathrm{mg} / \mathrm{ml}$ for the pure drug. Thus, there was a significant improvement in solubility $(P<0.01)$.

Flowability was assessed in terms of angle of repose, Carr's index and Hausnar ratio. The agglomerates had significantly lower angle of repose $(P<0.01)$ with the value in the range of $20.0-22.6^{0}$ as against $39.7^{0}$ for CFU, due to the irregular shape of the crystals, which facilitated the uniform flow of crystals from the funnel. Carr's index results reveal that flowability of CFU was also significantly poorer $(P<0.01)$ than those of the agglomerates with the latter having lower Carr index than pure CFU. Hausner ratio data similarly indicate that the agglomerates exhibited better flowability than the pure drug. The wettability test results in Table 1 indicate that the agglomerates were significantly $(P<$ 0.01 ) more wettable with a water-rising time of $6.9-8.0 \mathrm{~h}$ compared to $13.0 \mathrm{~h}$ for the pure drug The order of wettability was CFU-RLPO $>$ CFU-HPMC > CFU-HPC > CFU.

The in vitro drug release data, shown in Figure 1, revealed that drug release rates of the CFU agglomerates were largely similar but greater than that of the pure CFU. Powder X-ray diffraction patterns, displayed in Figure 2, indicate that pure CFU did not

Table 1: Physicochemical characteristics of CFU and recrystallized agglomerates $(n=3)$

\begin{tabular}{lcccc}
\hline \multicolumn{1}{c}{ Product code } & CFU & CFU-RLPO & CFU-HPMC & CFU-HPC \\
\hline Production yield $(\%)$ & ----- & $92.2 \pm 0.2$ & $88.6 \pm 0.2$ & $90.1 \pm 0.3$ \\
Solubility $(\mathrm{mg} / \mathrm{ml})$ & $0.30 \pm 0.27$ & $1.51 \pm 0.35$ & $1.33 \pm 0.15$ & $1.59 \pm 0.35$ \\
Content $(\%)$ & $97.0 \pm 0.6$ & $95.0 \pm 0.5$ & $94.0 \pm 0.6$ & $95.0 \pm 0.3$ \\
Solubility $(\mathrm{mg} / \mathrm{ml})$ & $0.16 \pm 0.03$ & $0.15 \pm 0.02$ & $0.31 \pm 0.03$ & $0.34 \pm 0.02$ \\
Water-rising time $(\mathrm{h})$ & $13.0 \pm 0.6$ & $6.9 \pm 0.6$ & $7.0 \pm 0.3$ & $8.0 \pm 0.2$ \\
Angle of Repose $\left(^{0}\right)$ & $39.7 \pm 0.3$ & $20.0 \pm 0.3$ & $22.6 \pm 0.2$ & $21.4 \pm 0.3$ \\
Carr's Index & $29.9 \pm 0.5$ & $15.8 \pm 1.0$ & $11.8 \pm 0.4$ & $15.6 \pm 0.4$ \\
Hausner Ratio & $1.29 \pm 0.63$ & $1.12 \pm 0.54$ & $1.07 \pm 0.29$ & $1.10 \pm 0.38$ \\
\hline
\end{tabular}




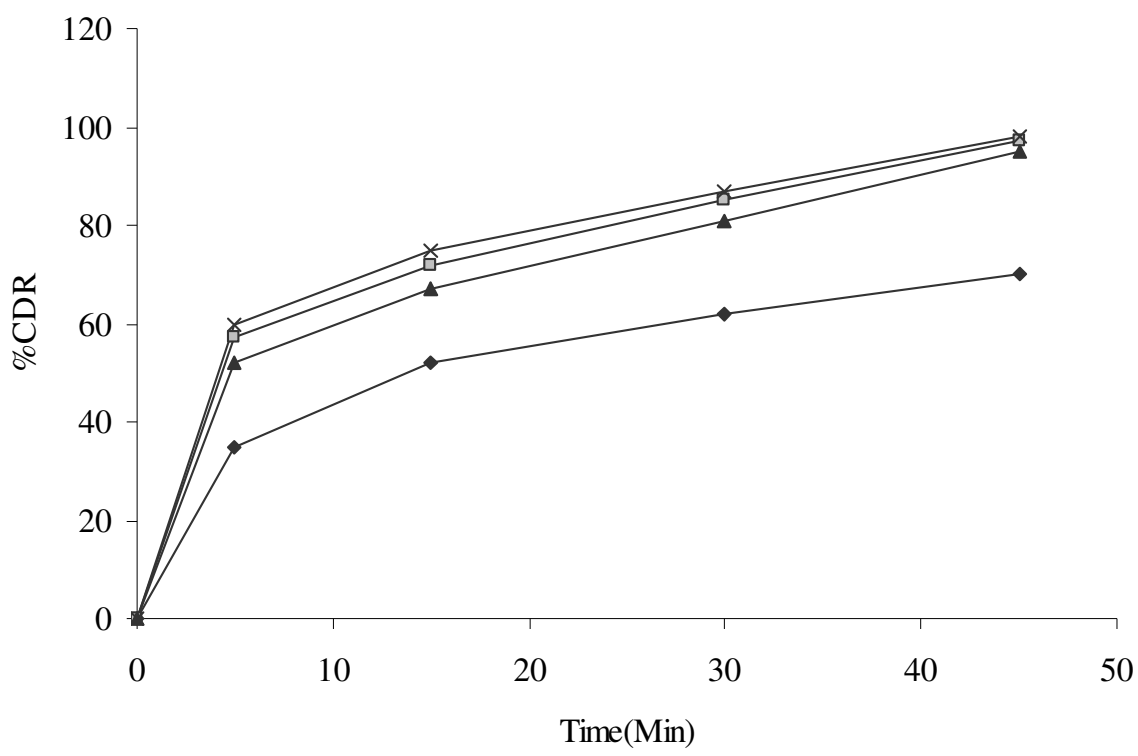

Figure 1: In vitro release profile of CFU and CFU agglomerates $(\rightarrow \mathrm{CFU}$; $\rightarrow$ - CFU-RLPO ; $\rightarrow$ CFU-HPMC; $\rightarrow$ CFU-HPC)

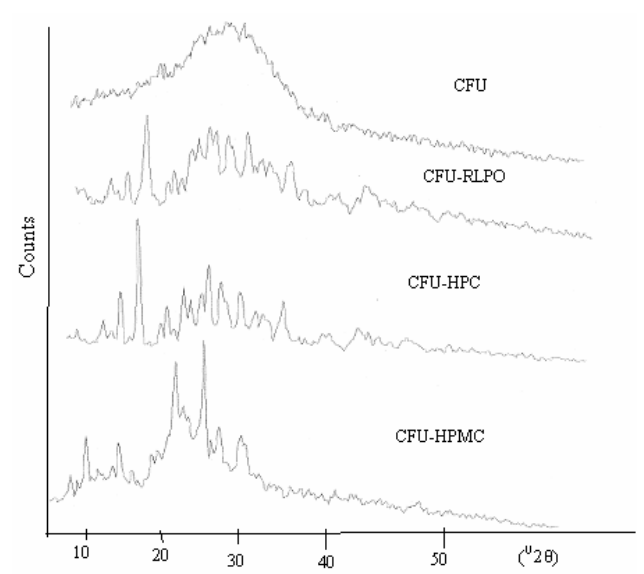

Figure 2: X-ray powder diffraction patterns of CFU and CFU agglomerates

show any peak suggesting that it is amorphous. The XRPD pattern of the agglomerates differed in the characteristic peak for cefuroxime axetil from that of the pure drug, thus confirming the existence of new compounds. There was a difference in $d-$ values (i.e., the distance between the successive two atomic planes of the crystals) between the XRPD spectrum of CFU and those of the agglomerates. For the DSC results (see Figure 3), the untreated drug showed a small endothermic diffuse peak at $212{ }^{0} \mathrm{C}$ corresponding to its fusion temperature, and thus, melting point. The corresponding endothermic peaks for CFURLPO, CFU-HPC and CFU-HPMC occurred at 207,211 and $208{ }^{\circ} \mathrm{C}$, respectively.

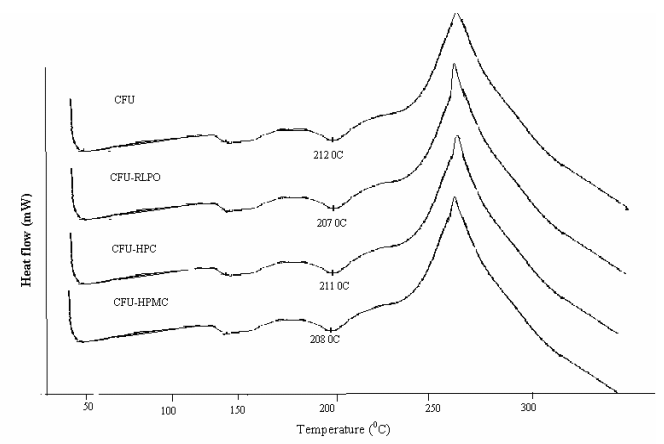

Figure 3: DSC thermograms of CFU and CFU agglomerates

The FTIR results are illustrated in Figure 4 and they reveal that the peak at $1685.34 \mathrm{~cm}^{-}$ ${ }^{1}$, corresponding to an amide group usually at $1695.34 \mathrm{~cm}^{-1}$ to $1630 \mathrm{~cm}^{-1}$, occurred at a higher wave number. For the agglomerates, there is a shift to a lower frequency with 
change in intensity, suggesting a change in the environment of the $\mathrm{C}=\mathrm{O}$ group associated with the amide moiety. The presence of absorption bands at $1249 \mathrm{~cm}^{-1}$, indicating aromatic ether in the drug and agglomerates and this suggests that there is no change in the environment of this moiety. The absorption bands at $1985.34 \mathrm{~cm}^{-1}$ and $1772.64 \mathrm{~cm}^{-1}$ are attributed to stretching vibration of the amide carbonyl in the ester and carboxylic acid.

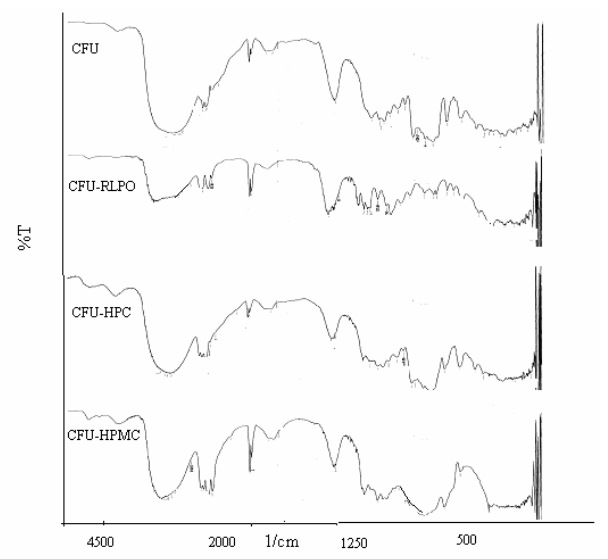

Figure 4: FTIR spectra of CFU and CFU agglomerates

\section{DISCUSSION}

The method used for the preparation of the CFU agglomerates was emulsion solvent diffusion (ESD) technique. In this method, when bridging liquid plus good solvent solution of the drug, along with different polymers, were poured into the poor solvent (dispersion medium) under agitation, quasiemulsion droplets of bridging liquid or good solvent form the emulsion droplet in the dispersing medium and induces the crystallization of the drug followed by agglomeration. The continuous phase is a liquid in which the drug solution is immiscible, and crystallization occurs inside the droplets because of counter diffusion of solvents through the droplets. The average diameter of the agglomerate crystals increased with increasing content of dichloromethane (DCM) in the system due to the enhanced agglomeration of powdery crystals. When the amount of acetone in the system was increased, while keeping the amount of DCM constant, the diffusion rate of acetone and DCM from the droplets was enhanced with increase in the content of acetone in the system. The increase in diffusion rate of DCM from the droplets shortened the agglomeration process of the crystals produced in the droplets while decrease in the DCM content of droplets reduced the agglomeration force of the crystal due to the increase in the unwetted part of the crystals with DCM in the agglomerates resulting in the formation of flocks with pendular bridges of water. Thus, the particle diameter and recovery of agglomerates decreased with increase in the acetone content of the system.

\section{Physicochemical properties}

The presence of the polymer, Eudragit, in CFU-RLPO enhanced the ability of the compound to form micelles, due to the presence of quaternary groups in the polymer, and thus increased the solubility of CFU. On the other hand, the presence of HPMC and HPC on the CFU particle surface would increase particle-particle interaction, thus causing faster squeezing out of DCM to the surface, and resulting in increased particle size and strength of agglomerates.

Furthermore, improvement in the solubility of the agglomerates may also be due to changes in the crystal forms as well as changes in crystal habit, structure and surface modification. In some instances, solvents incorporated into the crystals form solvets or clathrate that changes surface properties, reactivity of drug particles and the internal energy of the molecules, which play important roles in solubility improvement. The addition of HPMC and HPC, which are watersoluble polymers as stated earlier, increases the solubility of CFU in water by adsorbing on to the drug particle surface during agglomeration. 
The reason for the excellent flowability of the recrystallized agglomerates may be a reduction in interparticle friction as a result of probably a more spherical particle shape as well as larger crystal size. The rationale for the superior wettability of agglomerates is that the polymers adhere to the CFU crystals while their faster dissolution or release properties can be linked to their better wettability.

The agglomerates show slightly lower melting points than the pure drug but this may not be related to a change in the internal structure of the drug molecule. However, the change in the characteristic peaks and d-values can be linked to crystal habit modification while changes in the intensity of the peaks indicate a different arrangement of the molecules and hence suggest the development of new polymorphic forms.

In the FTIR study, the slight shift of the absorption band for the carbonyl group of the amide to a lower frequency can be attributed to the breakdown of the intermolecular hydrogen bond associated with the crystalline drug molecule and formation of hydrogen bond between the monomeric drug and the polymers. Overall, no changes occurred in the chemistry of CFU and there was no great fingerprint difference between CFU and the agglomerates.

\section{CONCLUSION}

The ESD technique used in this study produced agglomerates that exhibited significantly faster dissolution rates than the unmodified CFU crystals. The improved solubility and dissolution are attributable to the modification of CFU within the agglomerates due to specific interactions between the drug and the polymers, as well as increased wettability and a reduction in particle size. When this is taken together with improvements in other physicochemical properties, such as flowability and wettability, the ESD technique can be considered a suitable method for formulating CFU preparations with better physicochemical properties.

\section{ACKNOWLEDGEMENT}

The authors wish to thank Okasa Pharma, Satara (India) for providing cefuroxime axetil as a gift for this research work. The assistance of Mr Kalas of Shivaji university Kolhapur with XRD, DSC and FTIR studies is also acknowledged.

\section{REFERENCES}

1. Gibson M. Pharmaceutical Preformulation and Formulation - A Practical Guide from Candidate Drug Selection to Commercial Dosage Form. HIS Health Group, Englewood, 2001.

2. Kim ST, Kwon JH, Lee JJ, Kim CW. Microcrystallization of indomethacin using a pH-shift method. Int. J. Pharm, 2003; 263 (12): 141-150.

3. Chaumeil JC. Micronization: a method of improving the bioavailability of poorly soluble drugs. Meth. Find. Exp. Clin. Pharmacol, 1998; 20. 211-215.

4. Parrott EL, Comminution, in: Swarbrick J, Boylan JC (Eds.), Encyclopedia of Pharmaceutical Technology, vol. 3, Marcel Decker, New York, 1990, pp. 101-121.

5. Kaneniwa $N$, Ikekawa $A$. Influence of ball-milling atmosphere on decrease of molecular weight of polyvinylpyrrolidone powders. Chem. Pharm. Bull, 1972; 20: 1536-1543.

6. Waltersson JO, Lundgren P.The effect of mechanical comminution on drug stability.Acta Pharm. Suec, 1985; 22:291-300.

7. Vidgren MT, Vidgren PA, Paronen TP.Comparison of physical and inhalation properties of spraydried and mechanically micronized disodium cromoglycate. Int. J. Pharm, 1987; 35:139144.

8. Chawla KMG, Taylor JM, Newton MRC. Production of spray-ried salbutamol sulphate for use in dry powder aerosol formulations. Int. J. Pharm, 1994; 108: 233-240.

9. Steckel H. Rasenack N, Muller BW. In-situmicronization of disodiumcromoglycate for pulmonary delivery. Eur. J. Pharm. Biopharm, 2003; 55 (2):173-180.

10. Kawashima Y, Cui F, Takeuchi H, Niwa T, Hino T, Kiuchi $K$. Improvements in flowability and compressibility of pharmaceutical crystals for direct tabletting by spherical crystallization with a two-solvent system. Powder Technol, 1994b; 78: 151-157.

11. Kawashima $Y$, Handa T, Takeuchi H, Okumura M,Katou H, Nagata O.Crystal modification of 
phenytoin with polyethylene glycol for improving mechanical strength, dissolution rate and bioavailability by a spherical crystallization technique. Chem. Pharm. Bull, 1986; 34:3376-3383.

12. Sano A, Kuriki T, Kawashima $Y$, Takeuchi H, Hino $T$ , Niwa T,. Particle design of tolbutamide by spherical crystallization technique. $V$. Improvement of dissolution and bioavailability of direct compressed tablets prepared using tolbutamide agglomerated crystals.Chem. Pharm. Bull, 1992; 40:3030-3035.

13. Guillaume F. Guyot-Hermann AM Guyot JC.Spherical crystallization of meprobamate. II Farmaco, 1993; 48:473-485.

14. DiMartino $P$, Barthelemy C, Piva $F$, Joiris $E$, Palmieri GF, Martelli S.. Improved dissolution behavior of fenbufen by spherical crystallization. Drug Dev. Ind.Pharm, 1999; 25: 1073-1081.

15. Akbuga J.Preparation and evaluation of controlled release furosemide microspheres by spherical crystallization.Int. J. Pharm, 1989; 53: 99-105.
16. Ribardiere A, Tchoreloff $P$, Couarraze G, Puisieux F. Modification of ketoprofen bead structure produced by the spherical crystallization technique with a two-solvent system. Int. J. Pharm, 1996; 144:195-207.

17. Powell DA, James NC, Ossi MJ, Nahata MC, Donn $K H$.Pharmacokinetics of cefuroxime axetil suspension in infants and children. Antimicrobial. Agents. Chemother, 1991; 35: 2042-2045.

18. Woo JS, Chang HC. Non-crystalline cefuroxime axetil solid dispersant, process for preparing same and composition for oral administration thereof, 2000; US Patent 6107290.

19. Martino $P D$, Cristofaro RD, Barthelemy $C$, Joiris $E$, Filippo GP , Sante M. Improved compression properties of propyphenazone spherical crystals, Int J. Pharm, 2000; 197: 95-106

20. United States Pharmacopoeia (USP) 30/NF 24. United States Pharmacopoeial Convention Inc. Rockville, USA,, 2007, p. 242 